\title{
Oromandibular dystonia: A dental approach
}

\author{
Laura Maestre-Ferrín ${ }^{1}$, Juan-Andrés Burguera ${ }^{2}$, María Peñarrocha-Diago ${ }^{3}$, Miguel Peñarrocha-Diago ${ }^{4}$ \\ ${ }^{1}$ Student of Master of Oral Surgery and Implantology. Valencia University Medical and Dental School \\ ${ }^{2}$ Doctor of Neurology. University Hospital La Fe, Valencia \\ ${ }^{3}$ Associate Professor of Oral Surgery. Master of Oral Surgery and Implantology. Valencia University Medical and Dental School \\ ${ }^{4}$ Professor of Oral Surgery. Director of the Master of Oral Surgery and Implantology. Valencia University Medical and Dental \\ School
}

Correspondence:

Clínicas odontológicas

Gascó Oliag 1

46021-Valencia (Spain)

miguel.penarrocha@uv.es

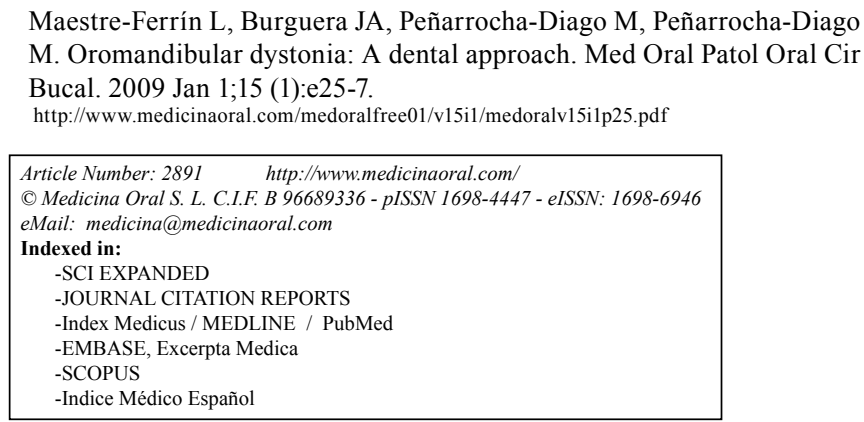

Received: 10/05/2009

Accepted: 02/08/2009

\begin{abstract}
Oromandibular dystonia consists of prolonged spasms of contraction of the muscles of the mouth and jaw. Primary idiopathic forms and secondary forms exist. Secondary dystonia develops due to environmental factors; some cases of cranial dystonia after dental procedure have been reported, but the causal relationship between these procedures and dystonia remains unclear. Traumatic situations in the mouth, such as poor aligned dentures or multiple teeth extractions may cause an impairment of proprioception of the oral cavity, leading to subsequent development of dystonia. The clinical characteristics of oromandibular dystonia are classified according to the affected muscles. The muscles involved may be the muscles of mastication, muscles of facial expression, or the muscles of the tongue. At present, there is no known cure for OMD. The mainstay of treatment for most focal dystonia is botulinum toxin injections. It is important for the dentist to be familiar with oromandibular dystonia, as it can develop after dental treatment and is often misdiagnosed as a dental problem.
\end{abstract}

Key words: Oromandibular dystonia, Meige's s syndrome.

\section{Introduction}

Oromandibular dystonia consists of prolonged spasms of contraction of the muscles of the mouth and jaw (1). It clinically manifests as sustained involuntary muscle contraction with twisting or repetitive movement or abnormal postures and a wide variation in clinical presentation, depending on musculature affected, severity, and distribution (2). In cranial dystonia, oromandibular dystonia occurs together with blepharospasm, this association is known as Meige's syndrome or Brueghel's syndrome (1).
The objective of this review is to carry out an update of this clinical entity, focusing on its dental implications.

\section{Classification and etiology}

Dystonia can be classified anatomically into regions of distribution such as focal, segmental, multifocal and generalized, and further into the affected body parts (2).

Another method for classifying dystonia is by etiology, into primary and secondary. Primary forms are also referred to as idiopathic, inherited or familial with genetic 
predisposition $(2,3)$. Secondary dystonia develops due to environmental factors: peripheral traumatic or surgical incidents, diseases of the brain (neurodegenerative disorders, cerebral infarction), and after treatment with neuroleptics or other medications (drug-induced dystonia) (4-6). The most common form of all secondary dystonia is tardive dystonia, which develops as a side effect of long-term treatment with antipsychotic drugs (2).

Tan and Jankovic conducted a clinical comparison between tardive and idiopathic oromandibular dystonia, they concluded that oro-facial-lingual stereotypes were significantly more frequent in the tardive than the idiopathic group. Dystonia in tardive oromandibular dystonia is more likely to be restricted to the oromandibular region, whereas in patients with idiopathic oromandibular dystonia, cervical dystonia often coexists (7).

Some cases of cranial dystonia after dental procedures have been reported, although the causal relationship between these procedures and dystonia is still unclear. Sankhla et al. (8) reported 27 peripherally induced OMD, four of which were wearing new sets of dentures, including one patient with an ill fitting dental bridge. The patients with ill fitting dentures had a history of using their jaw muscles to manipulate their jaw position to adapt to the new dentures. These malaligned dentures may have caused a proprioception impairment of the oral cavity leading to subsequent development of dystonia or so-called "edentulous dyskinesia". Hamzei et al. (9) reported the case of a woman with long-standing multiple system atrophy with predominance of parkinsonism who developed facial dystonia within a few hours and severe life-threatening laryngeal dystonia with respiratory failure within 3 days after insertion of ill-fitting dentures.

\section{Clinical Characteristics}

The clinical characteristics of oromandibular dystonia are classified according to the affected muscles. The muscles involved may be the muscles of mastication, muscles of facial expression, or the muscles of the tongue 10). Patients may present with jaw opening, jaw closing, jaw deflecting, jaw retruding dystonia, or a combination of any of these. The involuntary mandibular movements may be repetitive or sustained. Dystonic spasms may result in nasal contractions, facial grimacing, lip pursing, lip sucking, chewing, bruxism, tongue dyskinesias, retractions of corners of mouth, and platysma contractions. Other associated symptoms may include chewing difficulties, dysarthria, dysphagia, dysphonia, or breathing difficulties $(9,11)$.

Patients often report triggers such us stress, talking, chewing and praying (12). Often patients report that they have learned certain "sensory tricks" that help reduce the dystonia such as sleeping, relaxing, talking, singing, lip biting, tongue posturing, swallowing, chewing gum (12).

\section{Diagnosis}

The diagnosis of OMD is clinical and complicated, since it can present in various forms and with different degrees of severity. The differential diagnosis with TMJ disorders (such as bruxism or spontaneous condylar dislocation), hemifacial spasm and psychological disorders is necessary (10). Although this is an infrequent pathological entity, the dentist should be familiar with the symptomatology as it is often misdiagnosed as a "dental problem" and may cause considerable functional and psychosocial disability (8).

\section{Treatment}

At present there is no etiologic treatment for OMD (10). Oral medication is the usual first line of treatment. There is no evidence-based information about the efficacy of the different pharmacologic therapeutic options currently being applied in dystonia (13). Medications are prescribed in early stages and may have some effects in controlling dystonic movements. Systemic pharmacologic therapy benefits about one third of patients and consists of a wide variety of medications (cholinergics, benzodiazepams, antiparkinsonism drugs, anticonvulsants, baclofen, carbamazepine and lithium) (14). Although most oral medications have a low success rate, anticholinergic medications have been found to be the most effective oral medication for the treatment of dystonia (15).

Muscle afferent block (MAB), by intramuscular injection of anaesthetic and alcohol, has been used for the treatment of OMD. Yoshida et al. (16) studied the factors influencing this kind of treatment and concluded that MAB although is highly effective for OMD, it has no effect in patients with dyskinetic symptoms, thus OMD and oral/orofacial dyskinesia may have a different physiopathology.

The second line of treatment is botulinum toxin (BTX) injections, the mainstay of treatment for most focal dystonia. Botulinum toxin type A (BTX-A) blocks the release of acetylcholine at the presynaptic junction, producing a transient weakening of the muscle activity without systemic effects (17). Numerous studies have confirmed a $90-95 \%$ response rate to BTX injections (18). Bakke et al. (17) and Moller et al. $(3,19)$ reported a better effect of BTX injections when guided with electromyography (EMG), permitting individual treatment plans with longer intervals between BTX injections and lower doses than with conventional treatment for OMD. On the other hand, botulinum neurotoxins may be immunogenic, and some patients can develop secondary nonresponsiveness to BTX-A following multiple injections. Nevertheless, there have been only rare reports of patients with OMD treated with BTX-A becoming immuno-resistant. 
Operative therapies include peripheral and central nervous system procedures and are generally a last resort for individuals suffering with certain types of dystonia (10). In some patients with peripherally induced OMD, arthroscopy and other TMJ surgeries have been performed, although without obtaining satisfactory results, and in some cases the condition worsened (8). Yoshida (20) performed surgical resection of the bilateral coronoid processes for 2 patients with severe trismus due to jaw-closing dystonia, in whom treatment by botulinum toxin injection or muscle afferent block therapy had been ineffective. The jaw opening increased to $50 \mathrm{~mm}$ in both patients.

\section{Conclusion}

It is important for the dentist to be familiar with oromandibular dystonia, as it can develop after dental treatment, very often being misdiagnosed as a dental problem, and may cause considerable functional and psychosocial disability.

\section{References}

1. Marsden CD. Blepharospasm-oromandibular dystonia syndrome (Brueghel's syndrome). A variant of adult-onset torsion dystonia? J Neurol Neurosurg Psychiatry. 1976;39:1204-9.

2. Lee KH. Oromandibular dystonia. Oral Surg Oral Med Oral Pathol Oral Radiol Endod. 2007;104:491-6.

3. Møller E, Werdelin LM, Bakke M, Dalager T, Prytz S, Regeur L. Treatment of perioral dystonia with botulinum toxin in 4 cases of Meige's syndrome. Oral Surg Oral Med Oral Pathol Oral Radiol Endod. 2003;96:544-9.

4. Tan EK, Jankovic J. Bilateral hemifacial spasm: a report of five cases and a literature review. Mov Disord. 1999;14:345-9.

5. Yoshida Y, Akanuma J, Tochikubo S, Hoshi A, Matsuura Y, Homma M, et al. Slowly progressive dystonia following central pontine and extrapontine myelinolysis. Intern Med. 2000;39:956-60.
6. Tan EK, Chan LL, Wong MC. Levodopa-induced oromandibular dystonia in progressive supranuclear palsy. Clin Neurol Neurosurg. 2003;105:132-4

7. Tan EK, Jankovic J. Tardive and idiopathic oromandibular dystonia: a clinical comparison. J Neurol Neurosurg Psychiatry. 2000;68:186-90.

8. Sankhla C, Lai EC, Jankovic J. Peripherally induced oromandibular dystonia. J Neurol Neurosurg Psychiatry. 1998;65:722-8.

9. Hamzei F, Rijntjes M, Gbadamosi J, Fuchs K, Weiller C, Münchau A. Life-threatening respiratory failure due to cranial dystonia after dental procedure in a patient with multiple system atrophy. Mov Disord. 2003;18:959-61.

10. Balasubramaniam R, Rasmussen J, Carlson LW, Van Sickels JE, Okeson JP. Oromandibular dystonia revisited: a review and a unique case. J Oral Maxillofac Surg. 2008;66:379-86.

11. Grill S. Smothering dystonia in a patient with oromandibular dystonia. Mov Disord. 2004;19:1374-5.

12. Ilic TV, Pötter M, Holler I, Deuschl G, Volkmann J. Prayinginduced oromandibular dystonia. Mov Disord. 2005;20:385-6.

13. Balash Y, Giladi N. Efficacy of pharmacological treatment of dystonia: evidence-based review including meta-analysis of the effect of botulinum toxin and other cure options. Eur J Neurol. 2004;11:36170.

14. Goldman JG, Comella CL. Treatment of dystonia. Clin Neuropharmacol. 2003;26:102-8

15. Bressman SB. Dystonia update. Clin Neuropharmacol. 2000;23:239-51.

16. Yoshida K, Kaji R, Shibasaki H, Iizuka T. Factors influencing the therapeutic effect of muscle afferent block for oromandibular dystonia and dyskinesia: implications for their distinct pathophysiology. Int J Oral Maxillofac Surg. 2002;31:499-505.

17. Bakke M, Werdelin LM, Dalager T, Fuglsang-Frederiksen A, Prytz S, Møller E. Reduced jaw opening from paradoxical activity of mandibular elevator muscles treated with botulinum toxin. Eur J Neurol. 2003;10:695-9.

18. Dutton JJ. Botulinum-A toxin in the treatment of craniocervical muscle spasms: short- and long-term, local and systemic effects. Surv Ophthalmol. 1996;41:51-65.

19. Møller E, Bakke M, Dalager T, Werdelin LM. Oromandibular dystonia involving the lateral pterygoid muscles: four cases with different complexity. Mov Disord. 2007;22:785-90.

20. Yoshida K. Coronoidotomy as treatment for trismus due to jawclosing oromandibular dystonia. Mov Disord. 2006;21:1028-31. 